\title{
ULTRA HIGH SENSITIVITY GRAPHENE PIEZORESISTIVE PRESSURE SENSOR
}

\author{
H. Hosseinzadegan* , M. Pandey, and A. Lal
}

SonicMEM Lab., School of Electrical and Computer Engineering, Cornell University, Ithaca, NY, USA

\begin{abstract}
We demonstrate the first-ever pressure sensor utilizing graphene piezoresistors demonstrating the sensitivity as high as $323 \mu \mathrm{V} / \mathrm{V} / \mathrm{mmHg}$. This sensitivity is two orders of magnitudes higher than the commercial Omega PX140x series and 36 times higher than that of the reported carbon nanotube based transducers [1]. Our sensor consists of a $400 \mathrm{~nm} \mathrm{Si}_{\mathrm{x}} \mathrm{N}_{\mathrm{y}}$ membrane on silicon substrate with $10 \mathrm{~nm} / 140 \mathrm{~nm}$ thick $\mathrm{Cr} / \mathrm{Au}$ electrodes on top where the graphene layer is transferred on top of this structure. Our pressure sensor highly nonlinear, but can still be useful for applications where ultra-high sensitivity is needed and table lookup can be used.
\end{abstract}

\section{INTRODUCTION}

Piezoresistive pressure sensors are common devices used in biomedical to industrial monitoring applications. The small gaugefactor of the piezoresistive resistors, which are placed in a Wheatstone bridge configuration, limits the sensitivity of a given sensor. The gauge-factor, the membrane thickness, and the required sensitivity for a given application determine the pressure sensor membrane length, width, and thickness. A much higher gauge-factor and transducer sensitivity would enable a smaller sensor for constant sensitivity, or would enable a much more sensitive sensor for constant membrane area (Figure 1). Calculations in this figure are based on the equation (1) [2] for circular membrane pressure sensors where $a$ is the diameter, $h$ is the thickness, $G$ is the gauge-factor, $v$ is the Poisson's ratio, and $\mathrm{E}$ is the Young's modulus.

$$
\frac{\Delta V / V}{q}=G \frac{3}{8} \frac{\left(1-v^{2}\right)}{E}\left(\frac{a}{h}\right)^{2}
$$

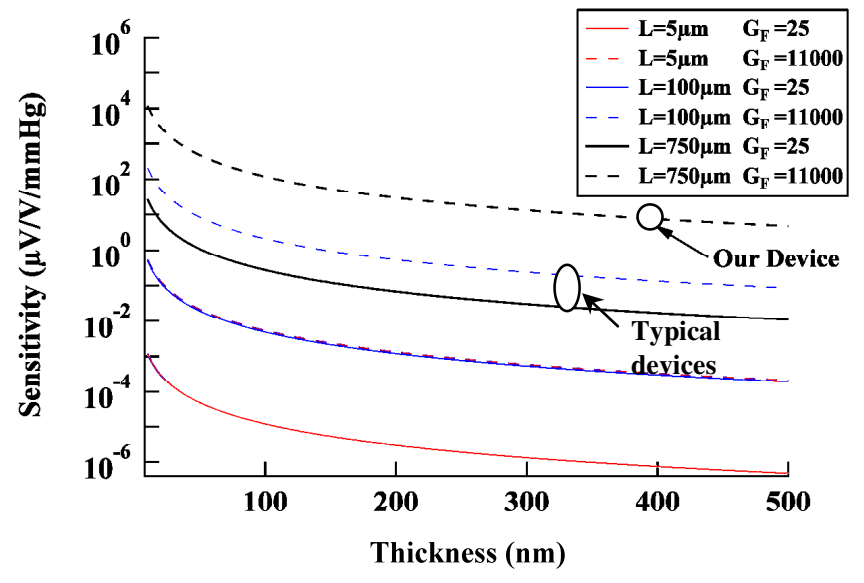

Figure 1: Relative sensitivity of strain transducers versus membrane thickness, for different lateral dimensions and gauge factor. Calculations

Given the $0.335 \mathrm{~nm}$ thickness of single or multi-layer graphene films, the overall reduction of the sensor is possible by reducing the membrane sizes to $100 \mathrm{~s}$ of $\mathrm{nm}$. Hence, graphene films may enable nano-scale pressure sensors that are more sensitive than existing polysilicon and silicon piezoreistor technologies, opening the pathways for using pressure sensors within biological cells, or forming high density pressure sensor arrays.
Tight binding theory for ideal graphene monolayers predicts a gauge factor of 2 for graphene nano ribbons and piezoresistance measurement via in-situ nanoindentation proves this estimation [3]. Similarly, the gauge factor about 11 was obtained at graphene/ epoxy composites cast in mold [4]. We have previously reported an ultrahigh piezo-resistivity coefficients [5]. In contrast to other reported value for graphene our samples are on silicon nitride films, which may result in charge modulation due to mechanical stresses. Another reason for the high value of piezoresistivity could be the grain boundary stretching in graphene films. Practical graphene film often consists of multiple layers of graphene sections with various crystalline grain sizes ranging from a few $\mathrm{nm}$ to 100 s of microns [6-7]. As a graphene film is stressed, the grain boundaries are likely to stretch modulating electron conduction along the boundaries.

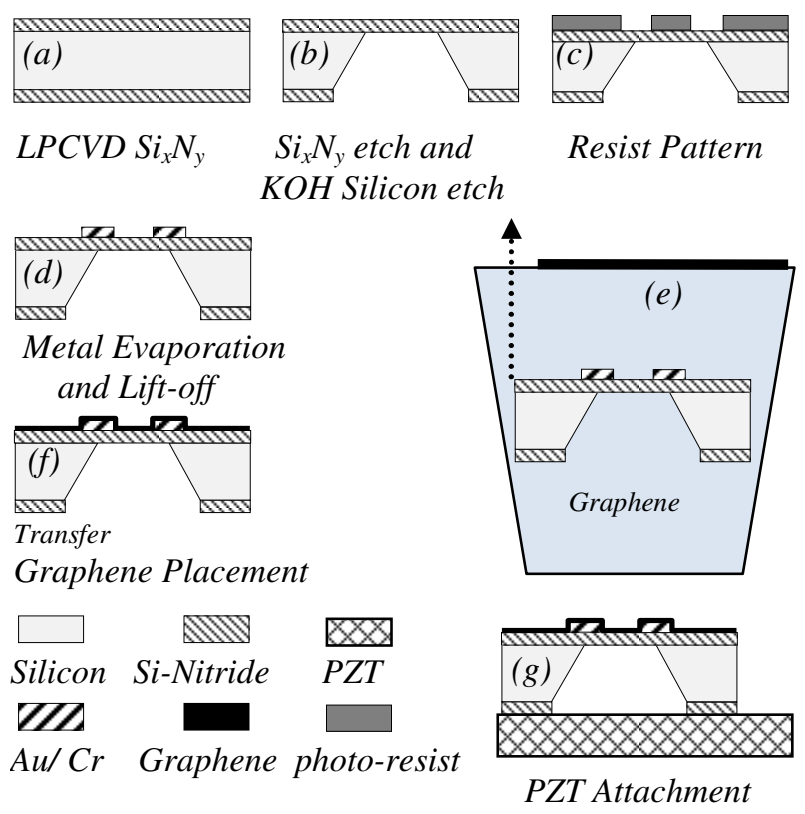

Figure 2. Process flow of graphene piezoresistive membrane type MEMS pressure sensor fabrication: Low stress LPCVD $S i_{x} N_{y}$ deposition (a), Patterning the silicon nitride film and wet etching of the silicon substrate using $\mathrm{KOH}$ solution (b), Patterning SPR220.3.0 photoresist (c), Cr/Au e-beam evaporation and lift-off $(d)$, Transferring graphene on top of the structure (e), Cleaning and drying the sample (f), and PZT attachment for ac actuation $(g)$.

Previous efforts have utilized electrodes or probes applied over the graphene films and then apply stress to the solid substrate to realize strain in the graphene films [8]. In this paper, we placed the graphene films over the electrodes defined over lows-stress $\mathrm{Si}_{\mathrm{x}} \mathrm{N}_{\mathrm{y}}$ membranes using a film transfer method. In this method, the graphene film is poly-crystalline with a grain sizes between 50$800 \mathrm{~nm}$, and can be two or three layers thick. The $\mathrm{Si}_{\mathrm{x}} \mathrm{N}_{\mathrm{y}} /$ electrode/graphene film stack membranes were actuated using a PZT actuator at resonance to obtain high strains, and the resulting change in resistance was used to measure the gauge factor [5]. Optical interferometer was used to measure the displacement 
as a result of PZT actuation, and the displacement was used to determine the strain. A very large gauge factor of 11289 was obtained. This is two to three orders of magnitude higher than that of most other materials which would enable piezoresistive transducer SNR to be higher by the same factor, making piezoresistive transducers much more attractive than other transduction mechanisms such as electrostatic or piezoelectric sense transducers. For example, for electrostatic transduction, the high sensitivity often occurs by decreasing gaps, but that often occurs at the cost of dynamic range and linearity. Piezoelectric films often require thicker films to obtain higher charge for a given film thickness, and do not scale well at the nanoscale.

\section{DEVICE FABRICATION}

Our device fabrication (Figure 2) starts with formation of a $400 \mathrm{~nm}$ low-stress LPCVD silicon-nitride on a 4-inch <100> ptype silicon substrate followed by anisotropic etching of the silicon substrate. A stack of $\mathrm{Cr}(10 \mathrm{~nm}) / \mathrm{Au}(140 \mathrm{~nm})$ is then evaporated and patterned using thermal evaporation to form a four-point probe structure. This structure consists of four wires on each side of the membrane converging from gaps of $10 \mu \mathrm{m}$ to $1 \mu \mathrm{m}$. A $0.5 \mu \mathrm{m}$ thick $\mathrm{SiO}_{2}$ film is sputtered and patterned over the electrodes so only the end section of electrodes are exposed. A CVD deposited [9] graphene-on-copper layer is transferred on top of the electrodes. The process of graphene transfer involves spinning a thin $(300 \mathrm{~nm})$ PMMA on top of graphene followed by wet etching of the copper foil. The silicon wafer which includes the nitride membranes is used to lift the PMMA/graphene bilayer floating on top of the $\mathrm{Cu}$ etch beaker. Acetone is used to remove the PMMA and samples are cleaned within DI water and let to dry for 6 hours. A PZT plate is adhesively attached to the back-side of the silicon die, with two wires soldered to the PZT plate. The Raman spectrum of the graphene films indicates a high quality film owing to the large Gto-D ratio. The D map is associated with defects in graphene [9]. The sheet resistance of the graphene film is $765-1500 \Omega / \square$, measured over 10 samples.

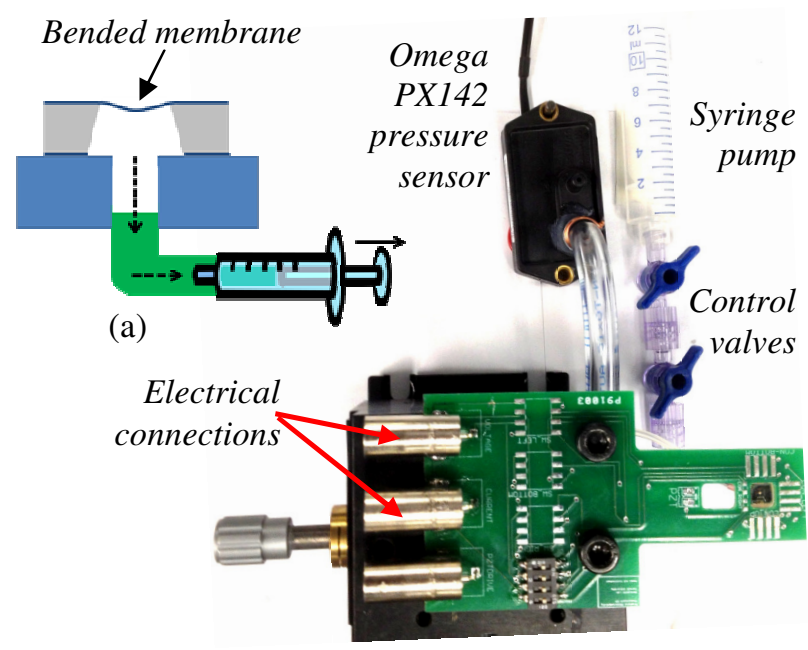

(b)

Figure 3: Schematic (a) and image (b) of measurement system including syringe pump, strain gauge, and leveling stage, and commercial pressure for calibration.

\section{PRESSURE SENSITIVITY MEASUREMENTS}

Measurement system consists of a syringe pump in series with two controlling valves, attached to the backside of the cavity and used to induce constant strain on graphene/ $\mathrm{Si}_{\mathrm{x}} \mathrm{N}_{\mathrm{y}}$ composite plate (Figure 3).
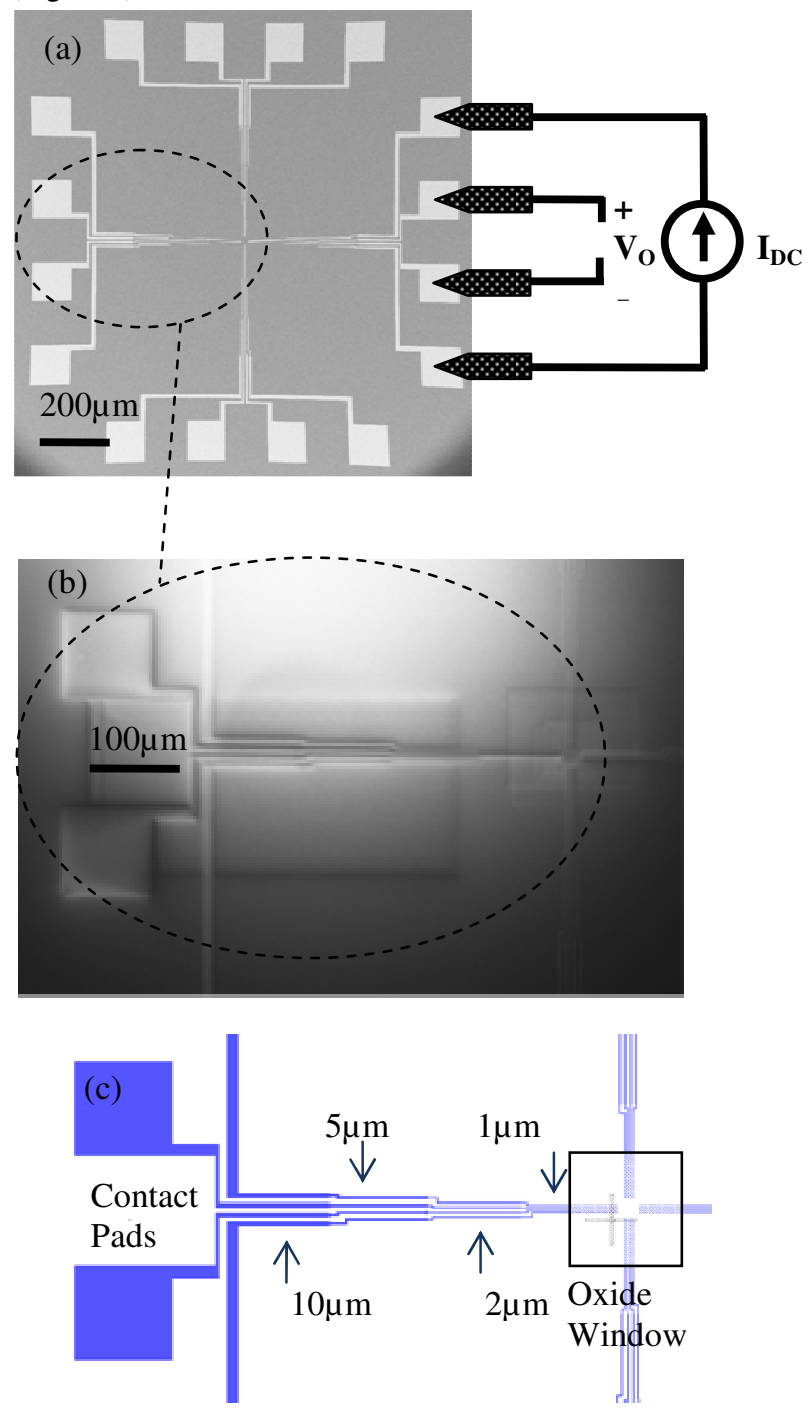

Figure 4: SEM image of 4-point probe electrodes on a silicon nitride membrane to measure pressure response from graphene layer placed in the oxide box ( $a$ and $b$ ), and schematic of this structure is in (c).

Pushing and pulling the pump induces tensile and compressive strains on the graphene/nitride film respectively. A printed circuit board (PCB) is designed to hold our device and carry electrical signals for PZT actuation and resistivity measurements. Entire PCB is mounted on a leveling stage with 0.1 degrees precision in order to make the membrane parallel to the Zygo 7300 optical displacement measurement system. Changes in graphene film resistance are monitored under this steady state strain by measuring the output voltage across the inner electrodes as a function of the applied DC current from the outer two electrodes (Figure 4).

\section{PRESSURE INDUCED STRESS SIMULATION}

Simulations are performed using finite element package ABAQUS for the entire PZT/Silicon/Membrane structure. Plane strain analysis is used here due to the relatively large lateral dimensions of the structure, compared to its thickness, which gives 


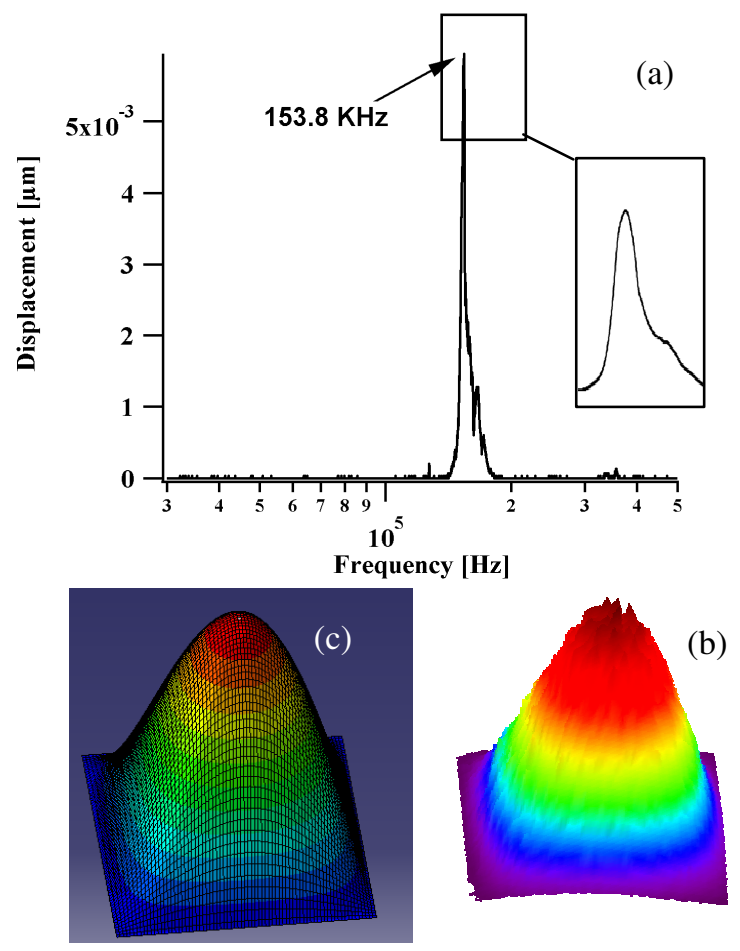

Figure 5. Displacement as a function of frequency at the center of the membrane where the piezoresistivity measuring 4-point probe is placed. (a). The first resonant frequency is at $153.8 \mathrm{KHz}$ with a quality factor of $80.2 \mathrm{in}$ air. Peak-to-peak voltage for the PZT actuation is $100 \mathrm{mV}$. The measured $(b)$ and simulated (c) mode shape of this membrane.

good agreement with preliminary 3D simulations.- The mode shape was obtained with assumed in-plane stress in the nitride membrane. A stress of $220 \mathrm{MPa}$ gives good agreement with the experiments and is within the expected range of stress obtained during fabrication. Steady state dynamic simulations were used to obtain resonance response of the structure. While nonlinear geometric effects are considered in the simulations, the PZT is modeled with a built-in linear model.

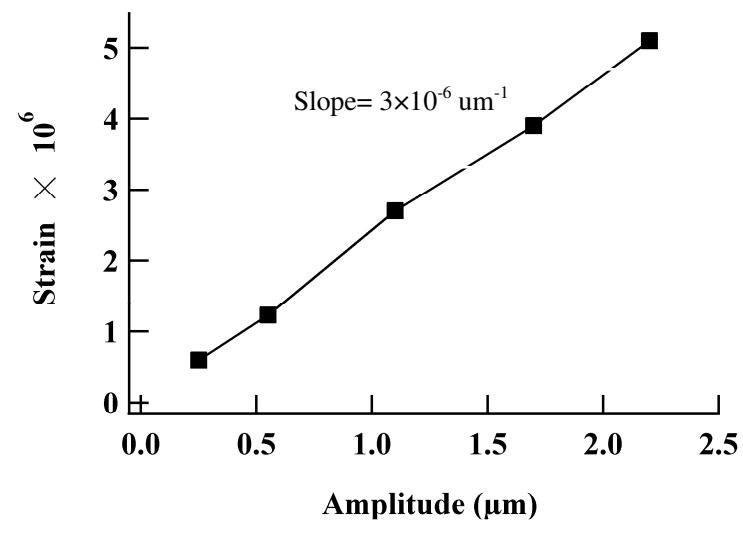

Figure 6. Simulated strain values at different amplitudes shows expected linear relationship

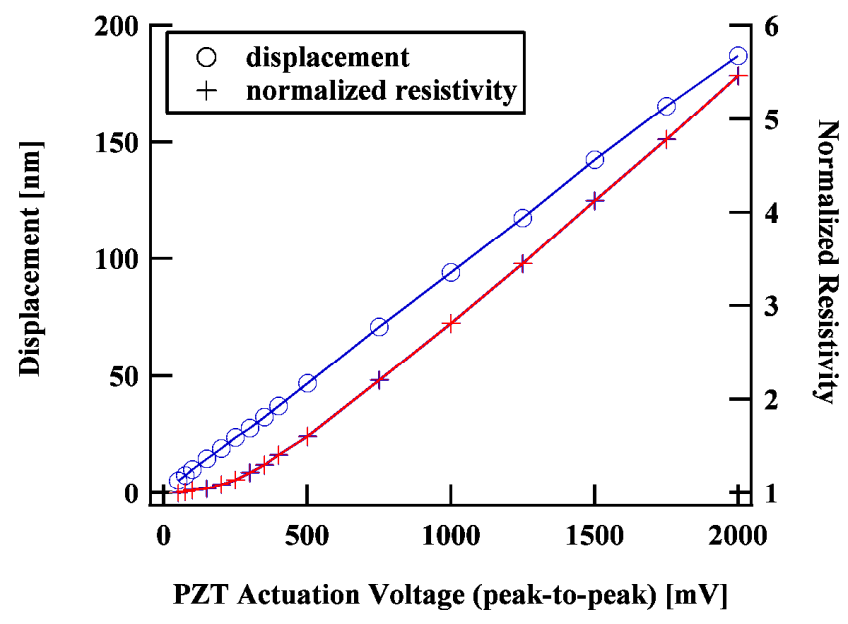

Figure 7. Blue curve is normalized resistivity (resistivity divided by maximum resistivity) as a function of drive amplitude. Red shows displacement at electrode location as a function of drive voltage, from which strain can be calculated.

The experimentally measured value of the quality factor is 80.2 (Figure 5-a), and is used in the simulations. The ABAQUS calculated displacement is in the order of $10 \mathrm{~nm}$ at resonance for a harmonic excitation of $1 \mathrm{~V}$ across PZT at the first resonance frequency. Corresponding strain values along the axis are in the order of $10^{-6}$. An expected linear dependence is obtained between the peak strain and amplitude of vibration (Figure 6). At the same time, the resistivity of the film can be measured as a function of applied PZT voltage (Figure 7). The PZT drive voltage was increased from $100 \mathrm{mV}_{\mathrm{pp}}$ to $10 \mathrm{~V}_{\mathrm{pp}}$.

The minimum limit of $50 \mathrm{mVpp}$ was imposed by the function generator HP-33120A. With this minimum drive voltage, the smallest AC strain that could be applied was $1.25 \times 10^{-6} \%$. Hence, our method is limited to measuring the gauge factors for strain range from $1.25 \times 10^{-6} \%$ to $2.5 \times 10^{-4} \%$. The $\mathrm{RF}$ coupling from the PZT drive to the four point probe was not responsible for the measurement of voltage across the 4-point probe, as the voltage across the inner electrodes also went to zero if the drive current was reduced to zero.

In order to calculate the gauge factor, the voltage between two inner electrodes is measured while actuating the PZT at resonant mode of the membrane with different amplitudes. Corresponding strain at each point is estimated using the linear relationship we came up using ABAQUS simulations (Figure 8).

\section{EXPERIMENTAL RESULTS}

The resistivity increases at both compressive and tensile strains which shows the graphene bends the same way regardless of the pressure direction in our present sensor configuration. Optical interferometer measurement of membrane displacements (Figure 9) indicates that the center part of the membrane moves similarly at positive and negative pressures due to pre-stress from $\mathrm{SiO}_{2}$ layer. The measured graphene gauge factor is orders of magnitude higher than that of most piezoresistive materials used in MEMS such as doped polysilicon and metals. Doped polysilicon can have gauge factors on the order of 30-40, while metals have a gauge factor of 55 [10-11]. 


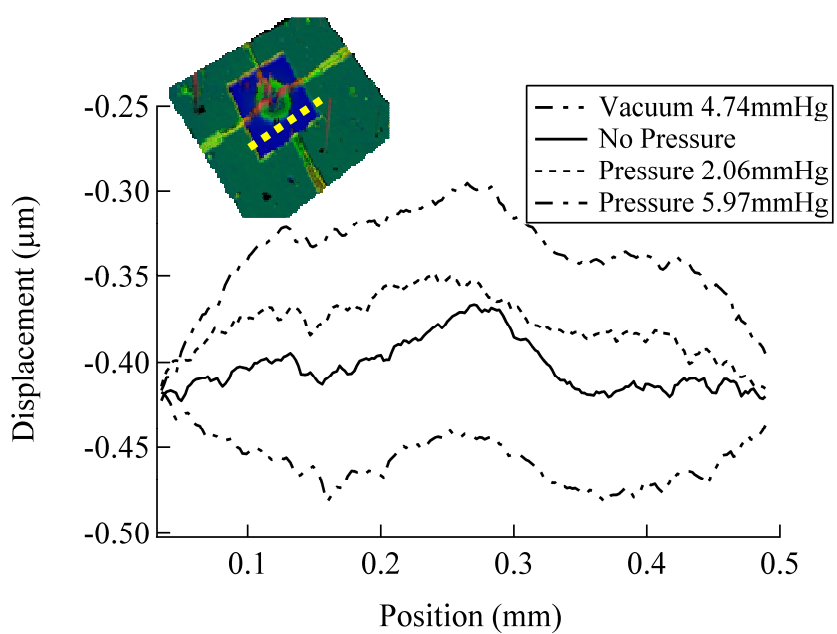

Figure 9: Zygo 7300 displacement profile along the shown the hatched line across the membrane. The dips show a pre-stressed nitride membrane due to the oxide film.

If the measured relative resistance is plotted against the estimated strain, the slope of the line is the gauge-factor (Figure 10).

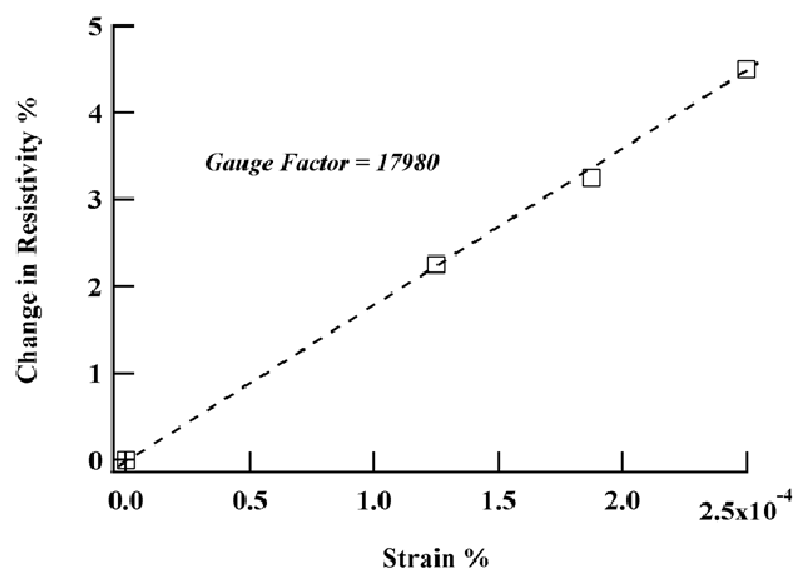

Figure 10. Normalized resistivity change versus applied AC strain at $153.8 \mathrm{kHz}$ membrane resonance.

Resistivity changes of the graphene film as a function of vacuum up to $5 \mathrm{mmHg}$ and positive pressures up to $6 \mathrm{mmHg}$ are plotted in Figure 11. The highly nonlinear behavior may be due to the fact that for either compressive or tensile stress the graphene membrane is stretched in the same direction. Another mechanism could be the generation of $\mathrm{Si}_{\mathrm{x}} \mathrm{N}_{\mathrm{y}}$ polarization charge is of the same variety whether the stress is compressive or tensile.

\section{CONCLUSION}

In summary, we present a pressure sensor process flow and results that pave the way for ultras-high sensitivity pressure sensors. Our estimated gauge-factor for this sensor is 11,300 matching that recently measured with $\mathrm{AC}$ resonance measurement of the graphene film [5]. However, we are exploring the exact reason for the nonlinearity, which maybe tunable for linear devices by adjusting the fabrication process.

\section{ACKNOWLEDGEMENTS}

We acknowledge support for this research from the DARPA TBN program. Travel support has been generously provided by the Transducer Research Foundation.

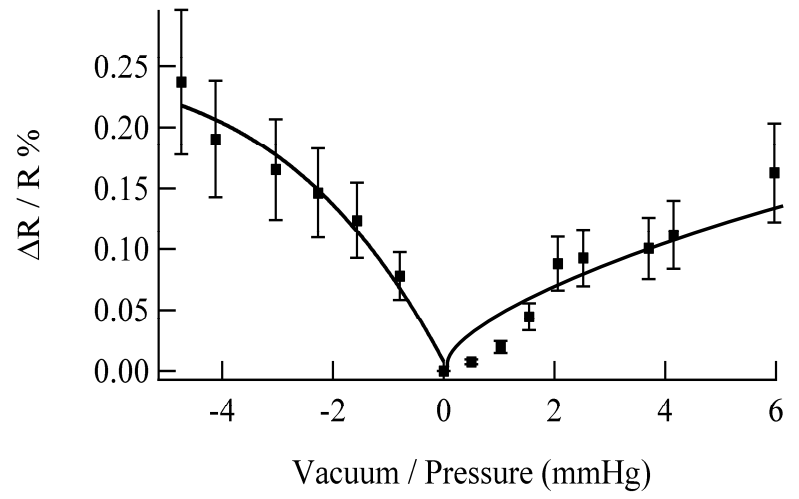

Figure 11: Resistivity change of graphene strain transducer as a function of pressure.

\section{REFERENCES}

[1] J. Cao, Q. Wang, and H. Dai, "Electromechanical Properties of Metallic, Quasimetallic, and Semiconducting Carbon. Nanotubes under Stretching", Physical Review Letter, 90, 157601-157604 (2003).

[2] L. Lin, H. Chu, and Y. Lu, "A Simulation Program for the Sensitivity and Linearity of Piezoresistive Pressure Sensors", Journal of Microelectromechanical Systems, 8, 514-522 (1999).

[3] M. Huang, and J. R. Greer, "Measuring Graphene Piezoresistance via in-situ Nanoindentation", ECS Transactions, Vol. 35 (3), pp. 211-216, 2011.

[4] Y. Kim et al. "Preparation of piezoresistive nano smart hybrid material based on graphene", Current Applied Physics, Vol. 11 (1), pp. S350-S352, 2010.

[5] H. Hosseinzadegan, C. Todd, A. Lal, M. Pandey, M. Levendorf, and J. Park, "Graphene Has Ultra High Piezoresistivie Gauge Factor", The $25^{\text {th }}$ IEEE International Conference on Micro Electro Mechanical Systems, Paris, France, (2012), pp. 611-614.

[6] P. Y. Huang, et al. "Grains and Grain Boundaries n Singlelayer Graphene Atomic Patchwork Quilts", Nature, Vol. 469, pp. 389-392, 2011.

[7] X. Li et al. "Graphene Films with Large Domain Size by a Two-step Chemical Vapor Deposition Process", Nano Lett., Vol. 10, pp. 4328-4334, 2010.

[8] A. B. Frazier and M. G. Allen, "Piezoreslstive Graphite/polyimide Thin Films for MicromachiningApplications", J. Appl. Phys., Vol. 73, 1993.

[9] Xuesong Li, et al., "Large-Area Synthesis of High-Quality and Uniform Graphene Films on Copper Foils", Science, 324, 1312-1314 (2009).

[10] M. le Berre, "Piezoresistive Properties of Boron-doped PECVD Micro- and Polycrystalline Silicon Films", Polycrystalline Thin Films Symposium, pp 733-8, 1994.

[11] M. Huth," Granular Metals: From Electronic Correlations to Strain-sensing Applications", J. of Applied Phys. ,Vol. 107, p.113709, 2010.

\section{CONTACT}

*H. Hosseinzadegan, tel: +1-607-255-1815; hh382@ cornell.edu 